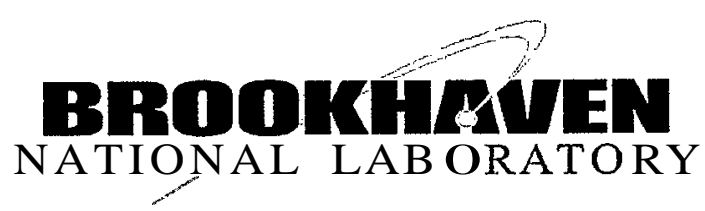

BNL-73491-2005-CP

\title{
Measurement and Correction of Nonlinear Chromaticity in RHIC
}

\author{
S. Tepikian, P. Cameron, A. Della Penna, V. Ptitsyn \\ Presented at the Particle Accelerator Conference (PAC'05) \\ Knoxville, Tennessee, \\ May 16-20,2005
}

\author{
Collider-Accelerator Department \\ Brookhaven National Laboratory \\ P.O. Box 5000 \\ Upton, NY I1973-5000 \\ www.bnl.gov \\ Managed by \\ Brookhaven Science Associates, LLC \\ for the United States Department: of Energy under \\ Contract No. DE-AC02-98CH10886
}

This is a preprint of a paper intended for publication in a journal or proceedings. Since changes may be made before publication, this preprint is made availablewith the understanding that it will not be cited Oreproducedwithout the permission of the author. 


\section{DISCLAIMER}

This report was prepared as an account of work sponsored by an agency of the United States Government. Neither the United States Government nor any agency thereof, nor any of their employees, nor any of their contractors, subcontractors, or their employees, makes any warranty, express or implied, or assumes any legal liability or responsibility for the accuracy, completeness, or any third party's use or the results of such use of any information, apparatus, product, or process disclosed, or represents that its use would not infringe privately owned rights. Reference herein to any specific commercial product, process, or service by trade name, trademark, manufacturer, or otherwise, does not necessarily constitute or imply its endorsement, recommendation, or favoring by the United States Government or any agency thereof or its contractors or subcontractors. The views and opinions of authors expressed herein do not necessarily state or reflect those of the United States Government or any agency thereof.

FOR UNCLASSIFIED, UNLIMITED STI PRODUCTS

Available electronically at:

OSTI:

\section{http://www.osti.gov/bridge}

Available for a processing fee to U.S. Department of Energy and its contractors, in paper from:

U.S. Department of Energy

Office of Scientific and Technical Information

P.O. Box 62

Oak Ridge, TN 37831

Phone: (865) 576-8401

Facsimile: (865) 576-5728

E-mail: reports@adonis.osti.gov

National Technical Information Service (NTIS):

Available for sale to the public from:

US. Department of Commerce

National Technical Information Service

5285 Port Royal Road

Springfield, VA 22131

Phone: (800) 553-6847

Facsimile: (703) 605-6900

Online ordering: http://www.ntis.gov/ordering.htm

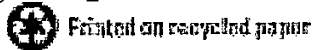




\title{
Measurement and Correction of Nonlinear Chromaticity in RHIC*
}

\author{
S. Tepikian, P. Cameron, A. Della Penna, V. Ptitsyn \\ Brookhaven National Lab, Upton, NY 11973, USA
}

\section{Abstract}

To improve luminosity in RHIC by using smaller $\beta^{*}$, higher order chromatic effects may need to be corrected [1]. Measuring of higher order chromaticities is discussed and compared to a model of RHIC, showing agreement. Assuming round beams, four families of octupoles are used to correct the second order chromaticities while keeping under control the amplitude dependent betatron tune spread in the beams. We show that the octupoles can reduce the second order chromaticity in RHIC, but they have insufficient strength for complete correction.

\section{THEORY}

Chromaticity is due to the tunes in an acceleratorchanging with the beams momentum [2]. This relationship can be expressed as:

$$
\mathrm{v}=\nu_{0}+\xi_{1} \delta+\xi_{2} \delta^{2} \ldots
$$

for either plane where $\delta=\Delta p / p, p$ is the longitudinal momentum of the beam, $\xi_{1}$ is the linear chromaticity, $\xi_{2}$ is the second order chromaticity, plus higher order terms. Here, one assumes that the beams motion is uncoupled in both planes.

Second order chromaticity can be changed with either sextupoles or octupoles. The next section describes the using four families of octupoles.

\section{Octupole Correction}

The contribution to the Hamiltonian due to the octupoles is:

$$
\mathrm{AH}=\left[\frac{1}{4} x^{4}-\frac{3}{2} x^{2} y^{2}+\frac{1}{4} y^{4}\right] b_{3}
$$

where $b_{3}$ is the strength of the octupole.

Applying the following change of variables to the Hamiltonian:

$$
x=\sqrt{2 J_{x} \beta_{x}} \cos \left(\phi_{x}\right)+\delta \eta_{x}
$$

and

$$
y=\sqrt{2 J_{y} \beta_{y}} \cos \left(\phi_{y}\right)+\delta \eta_{y}
$$

where $\eta_{(x, y)}$ are the dispersion functions, $\beta_{(x, y)}$ are the beta functions, $J_{(x, y)}$ are the actions and $\phi_{(x, y)}$ are the phases. The tune changes - to linear order in the octupole strength - are as follows:

$$
\Delta \nu_{(x, y)}=\frac{1}{2 \pi} \oint \frac{\partial \Delta H}{\partial J_{(x, y)}} d s
$$

\footnotetext{
${ }^{*}$ Work performed under Contract Number DE-AC02-98CH10886 with the auspices of the US Department of Energy.
}

This leads to (with $J=J_{\mathscr{T}}=J_{y}$ for round beams):

$$
\begin{aligned}
& \Delta \nu_{x}=\frac{1}{2 \pi}\left[\frac{3}{4} J\left\langle\left(\beta_{x}^{2}-2 \beta_{x} \beta_{y}\right) b_{3}\right\rangle+\frac{3}{2}\left\langle\beta_{x}\left(\eta_{x}^{2}-\eta_{y}^{2}\right) b_{3}\right\rangle \delta^{2}\right] \\
& \Delta \nu_{y}=\frac{1}{2 \pi}\left[\frac{3}{4} J\left(\left(\beta_{y}^{2}-2 \beta_{x} \beta_{y}\right) b_{3}\right\rangle+\frac{3}{2}\left\langle\beta_{y}\left(\eta_{y}^{2}-\eta_{x}^{2}\right) b_{3}\right) \delta^{2}\right]
\end{aligned}
$$

where $\langle\cdots\rangle$ denotes integration for one revolution and terms that have odd powers of the phase terms were assumed to cancel to zero.

Thus, the four families of octupoles satisfy the following four equations to introduce a change in the second order chromaticity:

$$
\begin{aligned}
\Delta \xi_{x_{2}} & =\frac{3}{4 \pi}\left\langle\beta_{x}\left(\eta_{x}^{2}-\eta_{y}^{2}\right) b_{3}\right\rangle \\
\Delta \xi_{y_{2}} & =\frac{3}{4 \pi}\left\langle\beta_{y}\left(\eta_{y}^{2}-\eta_{x}^{2}\right) b_{3}\right\rangle \\
0 & =\left\langle\left(\beta_{x}^{2}-2 \beta_{x} \beta_{y}\right) b_{3}\right\rangle \\
0 & =\left\langle\left(\beta_{y}^{2}-2 \beta_{x} \beta_{y}\right) b_{3}\right\rangle
\end{aligned}
$$

\section{MEASUREMENT}

RHIC consists of two rings named Blue and Yellow. In the Blue ring, the beam goes clockwise and for the Yellow ring, the beam is counter-clockwise. The chromaticites were measured in both rings. To measure the chromaticity, the tunes are measured at different momenta These tunes are then fit to a polynomial as a function of the momenta



Figure 1:A schematic for the changes to the radial steering during a measurement. A tune measurement is made at each plateau. The number of steps is adjustable. $\Delta r$ is the maximum size of the radial shift. 
Table 1: Second order chromaticity comparison

\begin{tabular}{|c|c|c|c|c|c|c|}
\hline Run\# & Ring & $\Delta r$ [mm] & X [meas] & X [model] & Y [meas] & Y [model] \\
\hline 4207 & Yellow & 2.0 & $12 \pm 26$ & 137 & $1332 \pm 28$ & 1141 \\
\hline & & & $-13 \pm 44$ & & $1661 \pm 119$ & \\
\hline & & & $94 \pm 27$ & & $1563 \pm 32$ & \\
\hline & & & $448 \pm 33$ & & $1452 \pm 25$ & \\
\hline 4454 & Blue & 2.0 & $410 \pm 19$ & 844 & $855 \pm 24$ & 940 \\
\hline & & & $346 \pm 27$ & & $838 \pm 62$ & \\
\hline & \multicolumn{7}{|c|}{ Averaging measured values for a comparison } \\
\hline & & $134 \pm 179$ & 137 & $1460 \pm 105$ & 1141 \\
\hline 4207 & Yellow & & $361 \pm 40$ & 844 & $870 \pm 26$ & 940 \\
\hline 4454 & Blue & & $292 \pm 31$ & & $924 \pm 48$ & \\
\hline
\end{tabular}

using linearregression. A statisticalerror is also computed. The momentum of the beam is changed by changing the frequency of the RF cavities. In RHIC, one can request a particular radial steering, the RF cavity frequencies are then changed to produce this radial shift. A schematic for radial steering is shown Fig. 1. For aperture reasons we have not used more than $2 \mathrm{~mm}$.

In a constantmagnetic field, the change in the momenta can be found from the radial shift using [2]:

$$
\frac{A R}{R}=\alpha \frac{A p}{P}
$$

where $\alpha=1 / \gamma_{T}^{2}$ is the momentum compaction factor and $\gamma_{T}$ is beam $\gamma$ at transition. For RHIC at store: $\gamma_{T}=23.32$.

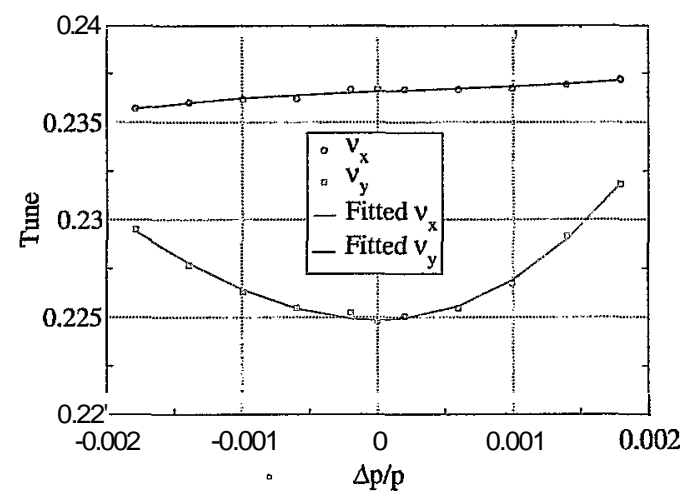

Figure 2 Tunes vs momentum with $\mathbf{1 1}$ data points in the Yellow ring. Fitting the data to a third order polynomial gives: $\xi_{x}=0.25, \xi_{x_{2}}=-49$ and $\xi_{y}=0.04, \xi_{y_{2}}=1800$. There is significant vertical $2^{\text {nd }}$ order chromaticity, but the horizontal is nearly linear.

The next section describes the tune-meter system.

\section{ThePLL TuneSystem [3]}

The tune-meter must have enough precision to be able to distinguish tune changes caused by the higher order chro- maticities. For this reason we chose the PLL tune-meter system which promises tune resolutions of $\delta \nu=0.0001$. Using a radial steering shift of $2 m m$, the $2^{\text {nd }}$ order chromaticity will shift the tune by almost 0.002 units as seen in Fig. 3.



Figure 3: The sensitivity of the tunes to changes in the radial steering. Note, for the third order chromaticity, the tune shift is too small to be measured for a normal RHIC store. We assumed $\xi_{2}=540$ and $\xi_{3}=67700$ for determining tune-meter requirements.

In the next section, we compare the measurements with the model.

\section{Comparison with Model}

To compare the measurements to the model, we use the design optics and adjust the tunes and linear chromaticities close to their measured values. The second order chromaticities are the calculated using MAD [4]. Here, we assume the major source of the $2^{\text {nd }}$ order chromaticity are the main quadrupoles and sextupoles. Some of the results are shown in Table 1. The largest difference is in the Blue ring's horizontal measurement. This difference could be due to the real optics being different from the design optics. Additionally, we have not taken into account other 
Table 2: Second order chromaticities with octupoles

\begin{tabular}{|r|r|r|r|r|r|r|} 
Case & octf (1) & octd (1) & octf (2) & octd (2) & $\boldsymbol{\xi}_{\mathbf{2}}$ & $\boldsymbol{\xi}_{\mathbf{2}}$ - \\
\hline 1 & 0 & 0 & 0 & 0 & -53 & 1953 \\
\hline 2 & 2 & 0 & 0 & 0 & -3 & 1739 \\
\hline 3 & 2 & -1 & 0 & 0 & -144 & 1670 \\
\hline 4 & 4 & -1 & 0 & 0 & 13 & 1445 \\
\hline 5 & 4 & -1 & 0 & 2 & -15 & 1802 \\
\hline 6 & 4 & 0 & -1 & -4 & 102 & 1696 \\
\hline 7 & 4 & 2 & -1 & -4 & 486 & 1575 \\
\hline 8 & 4 & 4 & -1 & -4 & 1029 & 1481 \\
\hline
\end{tabular}

sources of multipoles, orbit errors, coupling, etc.

\section{CORRECTION}

To correct the $2^{\text {nd }}$ order chromaticities, we use the four available families of octupoles in RHIC. Two of the families are in the arcs, while the othertwo are in low dispersion regions of the insertions. Table 3 shows the linear contribution of each octupole family to the $2^{\text {nd }}$ order chromaticities and tunes.

Table 3 Effect of the RHIC octupoles on $2^{\text {nd }}$ order chromaticity. There are four families of octupoles: (1) are in the arcs and (2) are in the insertions.

\begin{tabular}{|c|r|r|r|r|}
\hline & octf (1) & octd (1) & octf (2) & octd (2) \\
\hline$\Delta \xi_{x_{2}}$ & 165.9 & 11.7 & 2.4 & 0.3 \\
\hline$\Delta \xi_{y_{2}}$ & -37.0 & -47.4 & -0.6 & -0.5 \\
\hline$d \Delta \nu_{x} / d J$ & 388.5 & -288.5 & 135.8 & -105.9 \\
\hline$d \Delta \nu_{y} / d J$ & -278.4 & 339.2 & -111.4 & 127.6 \\
\hline
\end{tabular}

There is a maximum strength that the octupoles can be set (4 units). This limits the magnitude of the $2^{\text {nd }}$ order chromaticity that can be corrected. Table 2 shows the measured results when the octupoles are excited. From Table 3 , we can see that there is not enough strength in the octupoles to correct the vertical $2^{\text {nd }}$ order chromaticity and keep the tune spread minimized. However, we can reduce eliminate the horizontal and reduce the vertical $2^{\text {nd }}$ order chromaticity.

\section{SUMMARY}

The $2^{\text {nd }}$ order chromaticity was measured in both RHIC rings. Since the tune change is small, we needed a tunemeter with sufficient resolution. We chose to use the PLL tune-meter. In comparing the measured results with a RHIC model - except for one case - the results are near the model's, although, not within the error bars. Furthermore, the model used was the design model which may not represent the real machine.

The next step was to correct the $2^{\text {nd }}$ order chromaticity using four families of octupoles. Two of the families are used for the correction and the other two are used to reduce the amplitude dependent tune spreads (at least to linear order in the octupole strength). As shown, the octupoles can reduce the $2^{\text {nd }}$ order chromaticity, but there is insufficient 'strengthfor complete correction.

\section{REFERENCES}

[1] S. Tepikian, H. Huang, W. MacKay, F. Pilat, V. Ptitsyn, T. Satogata, D. Trbojevic, I. van Zeijts, "HighLuminosity $\beta^{*}=0.5 m$ RHIC Insertions", Particle AcceleratorConference (2003)

[2] M. Conte, W. W. MacKay, "AnIntroductionto the Physics of ParticleAccelerators", World Scientific Publishing Company (1991) and S. Y. Lee, "AcceleratorPhysics", World Scientific Publishing Company (1999).

[3] P. Cameron, J. Cupolo, W. Dawson, C. Degen, A. DellaPenna, M. Kesselman, A. Marusic, J. Mead, C. Schultheiss, R. Sikora, K. Vetter, "RHIC Third GenerationPLL Tune System", Particle AcceleratorConference (2003)

[4] F. Schmidt, "MAD-X Home Page", http://mad home.cern.ch/mad 